Published in final edited form as:

Science. 2006 September 29; 313(5795): 1922-1927. doi:10.1126/science.1132292.

\title{
Gene Regulatory Networks in the Evolution and Development of the Heart
}

\section{Eric N. Olson}

Department of Molecular Biology, University of Texas Southwestern Medical Center, 6000 Harry

Hines Boulevard, Dallas, TX 75390, USA. eric.olson@utsouthwestern.edu

\section{Abstract}

The heart, an ancient organ and the first to form and function during embryogenesis, evolved by the addition of new structures and functions to a primitive pump. Heart development is controlled by an evolutionarily conserved network of transcription factors that connect signaling pathways with genes for muscle growth, patterning, and contractility. During evolution, this ancestral gene network was expanded through gene duplication and co-option of additional networks. Mutations in components of the cardiac gene network cause congenital heart disease, the most common human birth defect. The consequences of such mutations reveal the logic of organogenesis and the evolutionary origins of morphological complexity.

The formation of organs and body parts proceeds by sequential gene regulatory steps that dictate cell fates and organize specialized cell types into complex three-dimensional units of structure and function. Studies of heart development and its genetic underpinnings in simple model organisms and in vertebrates have revealed an evolutionarily conserved gene regulatory network consisting of functional interconnections between myogenic transcription factors, their downstream target genes, and upstream signaling pathways that direct cardiac cell fate, myocyte differentiation, and cardiac morphogenesis $(1,2)$. Comparative genomic analyses of cardiac developmental control genes and their cisregulatory elements have also highlighted the conservation of genetic pathways that direct cardiogenesis.

The striking parallels between the transcriptional networks involved in heart development across vast phylogenetic distances support the idea that the evolutionary emergence of hearts with increasing complexity occurred through modification and expansion of an ancestral network of regulatory genes encoding cardiac transcription factors. The expansion of cardiac genetic networks through the duplication of cardiac regulatory genes and the co-option of additional gene networks probably allowed for the addition of new accessory structures, such as chambers, valves, and a conduction system, to a primitive vessel-like heart analogous to that of invertebrates and vertebrate embryos (3). The modular addition of innovations to primitive structures, although speculative, has also been proposed as a mechanism for the genesis of other vertebrate organs and body structures (4). Insights into the genetic circuits that drive the evolution and development of the heart shed light on general principles of organogenesis and evolutionary origins of morphological complexity, as well as the molecular basis of cardiovascular disease in humans. 


\section{Evolutionary Advancements of the Heart}

The most fundamental functional units of all hearts are cardiac muscle cells, which express an array of contractile proteins, such as muscle actin, myosin, troponin, and tropomyosin. The appearance of muscle cells preceded the divergence of Cnideria (hydra, jellyfish, and corals) and Ctenophora (comb jellies) from the Bilateria, from which mammals descended ( 700 million years ago) (Fig. 1) (5). Primordial muscle cells probably resembled the epithelio-muscle cells of Cnideria and amphioxus, which is thought to be the closest living approximation of the invertebrate ancestor of vertebrates (6-8). These cells probably existed in a primitive gastric pocket where they participated in fluid movement during feeding. Muscle cells in bilaterians are derived from mesoderm, which is believed to have arisen from the gastrodermis of a diploblastic ancestor. The diversification of muscle cells gave rise to skeletal, cardiac, and smooth muscle cells, and further specialization of cardiac muscle cells ultimately yielded atrial and ventricular myocytes, as well as the cells of the mammalian cardiac conduction system.

The first heart-like organ is believed to have appeared over 500 million years ago in an ancestral bilaterian $(6,9-11)$. It probably resembled the simple tubular vessel-like organs of tunicates and amphioxus, which contain a myoepithelial cell layer, lacking defined chambers or valves (Fig. 2). The heart of Drosophila, referred to as the dorsal vessel, also functions as a linear peristaltic pump but, in contrast to the hearts of tunicates and amphioxus, it ends in a closed cardiac compartment and contains a cardioaortic valve that separates a posterior lumen and an anterior aorta-like structure (Fig. 3) $(1,2)$. Nematodes do not possess a heart per se, but their pharynx contracts like a heart, and the muscle cells that line its walls exhibit electrical activity similar to that of mammalian cardiomyocytes (12).

During evolution, the heart evolved from a single-layered tube with peristaltic contractility to a more efficient and powerful pump with thick muscular chambers dedicated to receiving (atrial) and pumping (ventricular) blood, displaying synchronous contractions and seamless connections to a closed vascular system $(10,11)$. The transition from an aquatic to a terrestrial environment required several additional adaptations of the heart to separate oxygenated and deoxygenated blood (Fig. 2). The hearts of fish contain a single atrial chamber connected directly to a ventricle. Amphibians have two atria separated by a septum and a single ventricle. Terrestrial vertebrates have divided hearts in which septae separate the oxygenated and deoxygenated blood within the pulmonary and systemic circulations. Efficient unidirectional blood flow into and out of the heart was ensured by the appearance of valves. The conversion of a primitive heart tube to a multichambered heart that drives blood at high force through synchronous contractions also required a conduction system. Other advancements of the vertebrate heart include neural crest cells, which contribute to portions of the outflow tract and septum; trabeculae, which enhance oxygenation; the endothelium, which provides growth factor signals and precursor cells for formation of the cardiac valves; and the epicardium, which provides precursors for the coronary vasculature. 


\section{An Ancestral Genetic Network for Heart Development}

Heart development is governed by a core set of evolutionarily conserved transcription factors (NK2, MEF2, GATA, Tbx, and Hand) that controls cardiac cell fates, the expression of genes encoding contractile proteins, and the morphogenesis of cardiac structures (Fig. 1). These transcription factors also regulate each other's expression, serving to stabilize and reinforce the cardiac gene program (1,13-15). Dozens of other transcription factors contribute to cardiogenesis, in many cases by serving as accessory factors for these core regulators.

The MADS-box protein MEF2, which is conserved throughout the metazoans and exists even in yeast, is the most ancient myogenic transcription factor and presumably became irreversibly committed to the expression of muscle genes in an ancestral organism (16). As muscle cells diversified, MEF2 became a central component of muscle gene regulatory networks and is the only myogenic transcription factor known to be associated with the differentiation of all muscle cell types. In cardiac muscle cells, MEF2 cooperates with the core cardiac transcription factors to regulate contractile protein gene expression, whereas in skeletal muscle MEF2 cooperates with the MyoD family of bHLH transcription factors (16). Thus, MEF2 appears to have co-opted different transcriptional partners to regulate different muscle gene programs via specific combinations of cis-regulatory sequences.

Within cardiac muscle lineages, Mef2 fell under the control of NK2-type homeodomain proteins, which became dedicated to cardiac muscle and associated endodermal structures $(1,13,14,17)$. Mef2 and an NK2 homeobox gene closely related to those involved in the cardiac development of bilaterians are expressed in myoepithelial cells within the gastrodermis of Cniderians, which do not contain a heart (Fig. 1), suggesting that these genes were already associated with the muscle gene program in the common ancestor of these organisms (8). It has been postulated that a layer of muscle cells developing under the direction of an NK2-class gene within the endoderm of an ancestral organism, which may have been Cniderian-like, evolved into pulsatory muscular vessels of an early bilaterian (17).

The conservation of the core cardiac transcription factors and their cardiac expression in all modern-day organisms with hearts suggest that they became coupled to the expression of muscle genes involved in contractility and pump formation in an ancestral protochordate, and such regulatory interconnections were maintained and elaborated on during the evolution of more complex cardiac structures. Gene duplications during evolution increased the number of genes encoding these core cardiac transcription factors (Fig. 1). Such duplications, coupled with the modification of cis-regulatory elements, generated new patterns of gene expression; and variation in protein-coding regions conferred specialized activities, allowing the acquisition or modification of cardiac structures and functions. Consistent with the idea that new gene family members became more specialized and/or that functional redundancies masked their shared or subtle functions, mutations in cardiac regulatory genes in Drosophila, in which the cardiac regulatory network is relatively simple, often result in dramatic abnormalities in cardiac development, whereas mutations of individual paralogs of these genes in vertebrates frequently affect specific structures of the 
heart (such as ventricles or valves) that do not exist in the hearts of insects or more primitive organisms (1).

\section{Gene Networks in Drosophila Heart Development}

Drosophila has provided a powerful model for delineating the architecture of the cardiac regulatory network, due to the relative lack of functional redundancies in that network (Fig. 3). Formation of the dorsal vessel requires signaling by Decapentaplegic (Dpp), a member of the transforming growth factor- $\beta$ superfamily; fibroblast growth factor (FGF); and wingless (Wg), which belongs to the Wnt superfamily (1). The Drosophila NK2 homeobox gene, tinman, is essential for the specification of cardiac cell fates $(18,19)$ and serves as a target of inductive signals for cardiogenesis (Fig. 3). Among the target genes of tinman is the Mef2 gene, which is required for the differentiation of all types of muscles. Loss of function of the single Mef2 gene in Drosophila abolishes the expression of contractile protein genes in cardiac, skeletal, and visceral muscle cells but does not affect muscle cell identity, demonstrating the dedication of this factor to muscle differentiation.

Mutation of the GATA gene, pannier, in Drosophila results in an absence of cardioblasts and a decrease in the number of pericardial cells (20). tinman expression is lost in pannier mutants, and ectopic expression of pannier results in the production of supernumerary cardio-blasts. Multiple T-box genes function together with tinman and pannier to control cardiac fate, differentiation, and patterning of the dorsal vessel $(21,22)$. The Drosophila genome encodes a single member of the Hand family of bHLH transcription factors, which is directly regulated by Tinman and Pannier and is required for normal development of the dorsal vessel $(23,24)$. Autoregulatory and cross-regulatory interactions of tinman, Mef2, pannier, T-box, and Hand genes maintain the cardiac phenotype once the network has been activated by upstream inductive signals.

\section{Adding New Units of Structure and Function to the Vertebrate Heart}

In vertebrate embryos, cardiac precursor cells are specified in the lateral mesoderm by signals from adjacent tissues, many of which are conserved in organisms ranging from insects to mammals $(1,2)$. Cardiac progenitors from the primary heart field converge at the ventral midline to form a linear heart tube that resembles, both structurally and functionally, the primitive heart thought to exist in ancestral chordates. Development of the heart tube into the mature multichambered heart requires multiple steps that depend on genetic programs unique to vertebrates and/or amniotes. The heart tube gives rise to the left ventricle of the four-chambered heart, which is believed to represent the ancestral chordate cardiac compartment (13). The right ventricular chamber and outflow tract, later evolutionary advancements, are formed primarily from an adjacent population of precursors, referred to as the secondary or anterior heart field (25-27). Although there is debate as to whether this supplemental cell population represents a separate heart field versus an expanded region of the primary heart field, it is reasonable to conclude that the evolutionary addition of the right ventricle, as a new unit of cardiac structure and function, occurred through the recruitment of a novel population of precursor cells to a preexisting organ, rather than by simply expanding a common field of precursor cells. The hearts of fish and 
amphibians lack a right ventricle but contain a rudimentary outflow tract, a structure derived from the secondary heart field in mammals. It is currently unclear whether the outflow tract in these organisms is derived from the beginnings of a secondary heart field. It is also conceivable that the secondary heart field was first invented not to provide a new ventricle but simply to increase the mass of the original single ventricle, in which case it could have appeared in evolution before the second ventricle was invented.

At the posterior end of the heart tube, signaling by retinoic acid, a vertebrate invention, establishes atrial identity in cells that would otherwise adopt a ventricular fate (11), thereby conveying positional information along the anterior-posterior axis to chamber-specific genes. In this case, a new signaling pathway was coupled to the ancestral cardiac regulatory network to generate a new structure. Portions of the heart tube that do not become chamber myocardium give rise to the cardiac conduction system through genetic pathways that are also unique to vertebrates.

\section{Expansion of the Ancestral Cardiac Gene Network in Vertebrates}

Expansion of the number of ancestral cardiac regulatory genes (Fig. 1) and modification of the timing and pattern of their expression, as well as their regulatory interactions with each other and with other developmental control genes, was undoubtedly a major driving force for building cardiac complexity during evolution. Cardiac genes are typically controlled by combinations of cis-regulatory elements that operate in distinct regions of the heart (28). It is not unusual, for example, for separate enhancers to direct transcription of a gene in the right versus the left ventricle or even within subdomains of these structures that are otherwise indistinguishable. This diversity of regulatory elements has the potential to generate highly specialized groups of cells with distinctive gene expression profiles, allowing for the evolutionary modification of specific cardiac structures without affecting the entire organ. Such modularity in gene regulation is also revealed by the restriction of many cardiac defects to specific anatomical regions of the heart (14).

The homeobox gene $N k x 2-5$, a homolog of Drosophila tinman, is expressed in cardiac progenitor cells and the associated endoderm of all chordates, as well as urochordates and cephalochordates, and serves as a target of inductive signals that initiate cardiogenesis (7, 17, 28-30). Forced expression of Nkx2-5 in zebrafish or frog embryos expands the heart field and promotes cardiac gene expression $(31,32)$, whereas a dominant-negative $N k x 2-5$ mutant protein can block cardiogenesis in frog embryos; combined expression of $N k x 2-5$ and $N k x 2-3$ dominant-negative mutants results in a more severe phenotype than do mutants of either protein alone (33). The latter results have been interpreted as an indication of redundancy between these factors in the developing heart. However, overexpression of dominant-negative mutant proteins may also disrupt the activities of multiple transcription factors, particularly in the setting of heart development, in which transcription factors interact combinatorially, and may therefore result in more severe phenotypes than gene deletions. In mice lacking $N k x 2-5$, the initial events of heart formation occur normally, but embryos die from abnormal morphogenesis of the heart tube and failure in left ventricular development (34); defects that can be interpreted as a selective loss of derivatives of the primary heart field. The disparity between the essential early role of tinman in specification 
of the cardiac lineage in Drosophila and the relatively late cardiac defects in Nkx2-5 mutant mice could be explained if other NK2 homeo-domain proteins, or other cardiac transcription factors, substituted for an early function of Nkx2-5.

Of the four vertebrate $M e f 2$ genes, $M e f 2 c$ is required for activation of a subset of cardiac contractile protein genes, as well as for the development of cardiac structures derived from the secondary heart field, which are unique to amniotes (35). Thus, it appears that during evolution, this ancient myogenic regulator acquired new functions in regulating the formation of cardiac structures that occur only in more advanced hearts. Additional functions of vertebrate Mef 2 genes are likely to be masked by redundancies.

Members of the GATA family of zinc-finger transcription factors directly regulate numerous cardiac contractile protein genes, as well as upstream regulatory genes such as $N k x 2-5$, Mef2, and Hand (20). Of the six GATA genes in vertebrates, three (Gata4, -5 and -6 ) are expressed in the heart and have been implicated in heart development through loss-offunction mutations. Forced expression of cardiac GATA factors in Xenopus and zebrafish embryos induces premature activation of cardiac gene expression $(36,37)$.

At least seven Tbx genes display overlapping expression in the primary and secondary cardiac lineages and other cardiac structures of amniotes (38). Mice lacking Tbx5 display defects in the posterior region of the heart tube from which the atria are derived. Expression of a dominant-negative Tbx5 mutant in Xenopus embryos prevents formation of the primitive heart. $T b x 5$ and $T b x 20$ have also been implicated in formation of the cardiac conduction system and ventricular chambers, respectively. Tbx 2 and Tbx 3 function as repressors of chamber myocardium and are associated with the development of the conduction system (10).

The correlation between gene duplication and cardiac complexity is especially intriguing with respect to the Hand genes, which regulate ventricular growth $(13,14)$. Amphibians and fish, which contain only a single ventricle, express only one Hand gene, and zebrafish mutants lacking the Hand gene fail to form the ventricular chamber (39). In mice, Handl and Hand 2 are preferentially expressed in derivatives of the primary and secondary heart fields, respectively (13). Mice lacking Hand2 do not form a right ventricle, probably reflecting ablation of the secondary heart field (40), and Handl mutant embryonic stem cells are unable to contribute to the outer curvature of the heart that gives rise to the left ventricle (41). Deletion of Hand2 and $N k x 2-5$, which regulates Handl expression in the primary heart field, eliminates both ventricular chambers, leaving only an atrial remnant $(13,14)$. Thus, evolutionary duplication of the ventricular chambers correlates with duplication of the Hand genes.

\section{Building Cardiac Complexity by Co-opting New Genetic Networks}

The co-option of different upstream inputs by the core cardiac gene network appears to have played an important role in the evolution of the heart. In the tunicate Ciona, the single Mesp gene, encoding a bHLH transcription factor, is expressed in cardiac progenitors and is required for the expression of $N K 2$ and Hand genes $(29,42)$. Similarly, the two Mesp paralogs in the mouse (Mespl and -2) are redundantly required for formation of the cardiac 
mesoderm. In contrast, Mesp is not expressed in precursors of the Drosophila heart, suggesting that Mesp and its regulatory network were recruited to act upstream of the ancestral cardiac gene network during chordate evolution, or that the connection of Mesp to the cardiac network was lost during the evolution of insects.

Varying the upstream inputs to the cardiac regulatory network also provides an explanation for the development of the right ventricular chamber from the secondary heart field. Cardiac muscle cells in both the right and left ventricles rely on the same set of transcription factors for activation of the gene program for cardiomyocyte differentiation and the expression of con tractile protein genes, but the upstream inputs into this regulatory network differ in cells derived from the primary and secondary heart fields (Fig. 4A). The evolutionary addition of the secondary heart field required a signaling mechanism to activate the core cardiac transcriptional network. The Isl1 transcription factor, which is expressed specifically in the secondary heart field (26), directly activates the Mef2c gene in this population of cardiac precursor cells (Fig. 4B) (43). In this case, Isl1 was connected to the cardiac regulatory network, possibly through the acquisition of Isl1-dependent enhancer modules by Mef2c and perhaps other core cardiac regulatory genes. Because Isl1 is not cardiac-specific, its initial activation and its actions on downstream targets require combinatorial mechanisms with other factors or epigenetic influences. GATA factors and Nkx2-5, which are expressed in both heart fields and are required for $M e f 2 c$ expression in the secondary heart field, may serve this role. The forkhead transcription factor Foxh1 also activates Mef2c transcription in the secondary heart field through a separate enhancer and appears to act downstream of Isl1 (44). Mutations in Isll, Mef2c, or Foxhl all result in severe cardiac defects that appear to reflect ablation of the secondary heart field and its descendent structures $(26,35,44)$, demonstrating the interdependence of these cardiogenic regulators.

These findings suggest that the evolution of the four-chambered heart involved the acquisition of a new set of regulatory inputs into the ancestral cardiac transcription factor genes. Because genes within the core cardiac network cross-regulate and autoregulate their expression, activation of one or a few of the genes in the network may ultimately activate them all, as well as common sets of downstream genes. The regulation of the core set of myogenic transcription factors by different upstream signals in different cardiac muscle precursor populations is reminiscent of the strategy for skeletal muscle development in which members of the MyoD family are regulated by different upstream signals and transcriptional inputs in different skeletal muscle lineages, thereby contributing to muscle diversity and specialization (45).

\section{Downstream Targets of Cardiac Transcription Factors}

Relatively little is known about the downstream target genes of the core cardiac transcrip tion factors that drive cardiac growth and morphogenesis. How cardiac looping occurs and how the ventricular chambers adopt their specific shapes and positions are important unanswered questions. Morphogenesis and growth of the heart are intimately connected to cardiac function, but the mechanistic basis of this link is also vague, as are the mechanisms whereby the heart (or other organs) coordinates its size with that of the body. Molding of the cardiac chambers depends on myocyte differentiation and contract-ile activity as well as 
blood flow, fluid dynamics, and oxygenation. How these intrinsic and extrinsic influences govern the growth and development of the heart is unclear.

In addition to the structural and regulatory genes that control cardiac development and contractility, several evolutionarily conserved micro-RNAs (miRNAs), which function as negative regulators of target RNAs, are expressed specifically in the developing heart. One such miRNA, Mirl, negatively regulates cardiac growth during mouse development by inhibiting translation of Hand2 (46). In Drosophila, Mirl is required for proper patterning of the dorsal vessel, but the Drosophila Hand mRNA is not a target of Mirl, suggesting that other evolutionarily conserved targets may exist or that Mirl acts differently in insect and mammalian heart development (47). Understanding the roles of miRNAs in heart development and disease represents a rich area for future investigation.

\section{Insights into Human Heart Disease}

Heterozygous mutations in cardiac regulatory genes frequently cause congenital heart disease in humans, illustrating the exquisite sensitivity of cardiac structure and function to genetic perturbation. Mutations in Nkx2-5 cause a spectrum of congenital heart defects (48), including cardiac conduction abnormalities, ventricular-septal defects (VSDs), and atrialseptal defects (ASDs). Mutations in Tbx5 are responsible for Holt-Oram syndrome (49), an autosomal dominant disorder associated with structural and functional cardiac defects, and deletion of Tbx1 results in malformations of the cardiac outflow tract and VSDs due to failure in the migration of neural crest cells to the heart (50). Mutations in GATA4, some of which disrupt its interaction with Tbx5, cause ASDs and VSDs (51). The realization that heart defects in humans often result from haploinsufficiency of cardiac transcription factors suggests that strategies to enhance the activity of such developmental regulators, even subtly, may provide therapeutic benefit.

The discovery of cardiac regulatory gene networks has allowed for genetic testing for cardiac disease genes. However, congenital heart disease in humans commonly displays variable penetrance and expressivity, pointing to the influence of modifier genes and environmental influences on cardiac phenotypes. Understanding the molecular basis of such variability is an important challenge for the future.

Warm-blooded animals are unable to effectively repair the injured myocardium, whereas amphibians, fish, mollusks, and arthropods can replace lost cardiac myocytes through regeneration, suggesting that the ability to undergo cardiac regeneration may represent a primordial metazoan attribute that was lost. There is evidence for a contribution of stem cells to repair of the mammalian myocardium, but this endogenous mechanism is inadequate to restore function to the failing heart. In addition to their roles in heart formation, many developmental regulators, such as MEF2, GATA4, and Nkx2-5, are redeployed after injury to the adult heart and ensuing changes in cardiac contractility and function (52). There is great interest in therapeutically manipulating the activities of these transcription factors in the adult heart to promote cardiac repair, including the genesis of specialized cardiac cell types from stem cells for cellular replacement. 
Further analysis of the genetic networks that govern heart development through the combined use of genomics, genetics, and model organisms promises to yield insights, not only into general principles of organogenesis, but also to facilitate therapies for congenital and acquired heart disease.

\section{Acknowledgments}

Work in E. Olson's lab was supported by grants from NIH, the Donald W. Reynolds Center for Clinical Cardiovascular Research, and the Robert A. Welch Foundation. Input from numerous colleagues and members of the Olson lab is gratefully acknowledged.

\section{References and Notes}

1. Cripps RM, Olson EN. Dev. Biol. 1998; 203:106. [PubMed: 9806776]

2. Bodmer R. Trends Cardiovasc. Med. 1995; 5:21. [PubMed: 21232234]

3. Fishman MC, Olson EN. Cell. 1997; 91:153. [PubMed: 9346232]

4. Davidson, EH. Genomic Regulatory Systems. Vol. 1. Academic Press; San Diego, CA: 2001.

5. Oota S, Saitou N. Mol. Biol. Evol. 1999; 16:856. [PubMed: 10368962]

6. Bishopric NH. Ann. N. Y. Acad. Sci. 2005; 1047:13. [PubMed: 16093481]

7. Holland ND, Venkatesh TV, Holland LZ, Jacobs DK, Bodmer R. Dev. Biol. 2003; 255:128. [PubMed: 12618138]

8. Grens A, et al. Dev. Biol. 1996; 180:473. [PubMed: 8954720]

9. Romer AS. Science. 1967; 158:1629. [PubMed: 4862529]

10. Moorman AFM, Christoffels VM. Physiol. Rev. 2003; 83:1223. [PubMed: 14506305]

11. Simoes-Costa MS, et al. Dev. Biol. 2005; 277:1. [PubMed: 15572135]

12. Avery, L.; Thomas, JH. The Nematode C. elegans II. Riddle, D., editor. Cold Spring Harbor Laboratory Press; Cold Spring Harbor, NY: 1997. p. 679-716.

13. Buckingham M, Meilhac S, Zaffran S. Nat. Rev. Genet. 2005; 6:826. [PubMed: 16304598]

14. Srivastava D, Olson EN. Nature. 2000; 407:221. [PubMed: 11001064]

15. Bruneau BG. Circ. Res. 2002; 90:509. [PubMed: 11909814]

16. Black BL, Olson EN. Annu. Rev. Cell Dev. Biol. 1998; 14:167. [PubMed: 9891782]

17. Harvey RP. Dev. Biol. 1996; 178:203. [PubMed: 8812123]

18. Bodmer R. Development. 1993; 118:719. [PubMed: 7915669]

19. Azpiazu N, Frasch M. Genes Dev. 1993; 7:1325. [PubMed: 8101173]

20. Sorrentino RP, Gajewski KM, Schulz RA. Semin. Cell Dev. Biol. 2005; 16:107. [PubMed: 15659345]

21. Reim I, Mohler JP, Frasch M. Mech. Dev. 2005; 122:1056. [PubMed: 15922573]

22. Miskolczi-McCallum CM, Scavetta RJ, Svendsen PC, Soanes KH, Brook WJ. Dev. Biol. 2005; 278:459. [PubMed: 15680363]

23. Han Z, Yi P, Li X, Olson EN. Development. 2006; 133:1175. [PubMed: 16467358]

24. Kölsch V, Paululat A. Dev. Genes Evol. 2002; 212:473. [PubMed: 12424518]

25. Abu-Issa R, Waldo K, Kirby ML. Dev. Biol. 2004; 272:281. [PubMed: 15282148]

26. Cai CL, et al. Dev. Cell. 2003; 5:877. [PubMed: 14667410]

27. Kelly RG, Brown NA, Buckingham ME. Dev. Cell. 2001; 1:435. [PubMed: 11702954]

28. Schwartz RJ, Olson EN. Development. 1999; 126:4187. [PubMed: 10477287]

29. Satou Y, Imai KS, Satoh N. Development. 2004; 131:2533. [PubMed: 15115756]

30. Davidson B, Levine M. Proc. Natl. Acad. Sci. U.S.A. 2003; 100:11469. [PubMed: 14500781]

31. Chen JN, Fishman MC. Development. 1996; 122:3809. [PubMed: 9012502]

32. Cleaver OB, Patterson KD, Krieg PA. Development. 1996; 122:3549. [PubMed: 8951070]

33. Fu Y, Yan W, Mohun TJ, Evans SM. Development. 1998; 125:4439. [PubMed: 9778503] 
34. Lyons I, et al. Genes Dev. 1995; 9:1654. [PubMed: 7628699]

35. Lin Q, Schwarz J, Bucana C, Olson EN. Science. 1997; 276:1404. [PubMed: 9162005]

36. Reiter JF, et al. Genes Dev. 1999; 13:2983. [PubMed: 10580005]

37. Latinkic BV, Kotecha S, Mohun TJ. Development. 2003; 130:3865. [PubMed: 12835401]

38. Plageman TF Jr. Yutzey KE. Dev. Dyn. 2005; 232:11. [PubMed: 15580613]

39. Yelon D, et al. Development. 2000; 127:2573. [PubMed: 10821756]

40. Srivastava D, et al. Nat. Genet. 1997; 16:154. [PubMed: 9171826]

41. Riley PR, Gertsenstein M, Dawson K, Cross JC. Dev. Biol. 2000; 227:156. [PubMed: 11076684]

42. Davidson B, Shi W, Levine M. Development. 2005; 132:4811. [PubMed: 16207759]

43. Dodou E, Verzi MP, Anderson JP, Xu SM, Black BL. Development. 2004; 131:3931. [PubMed:

15253934]

44. von Both I, et al. Dev. Cell. 2004; 7:331. [PubMed: 15363409]

45. Pownall ME, Gustafsson MK, Emerson CP Jr. Annu. Rev. Cell Dev. Biol. 2002; 18:747. [PubMed: 12142270]

46. Zhao Y, Samal E, Srivastava D. Nature. 2005; 436:214. [PubMed: 15951802]

47. Kwon C, Han Z, Olson EN, Srivastava D. Proc. Natl. Acad. Sci. U.S.A. 2005; 102:18986. [PubMed: 16357195]

48. Basson CT, et al. Nat. Genet. 1997; 15:30. [PubMed: 8988165]

49. Lindsay EA, et al. Nature. 2001; 410:97. [PubMed: 11242049]

50. Merscher S, et al. Cell. 2001; 104:619. [PubMed: 11239417]

51. Garg V, et al. Nature. 2003; 424:443. [PubMed: 12845333]

52. Olson EN, Schneider MD. Genes Dev. 2003; 17:1937. [PubMed: 12893779] 


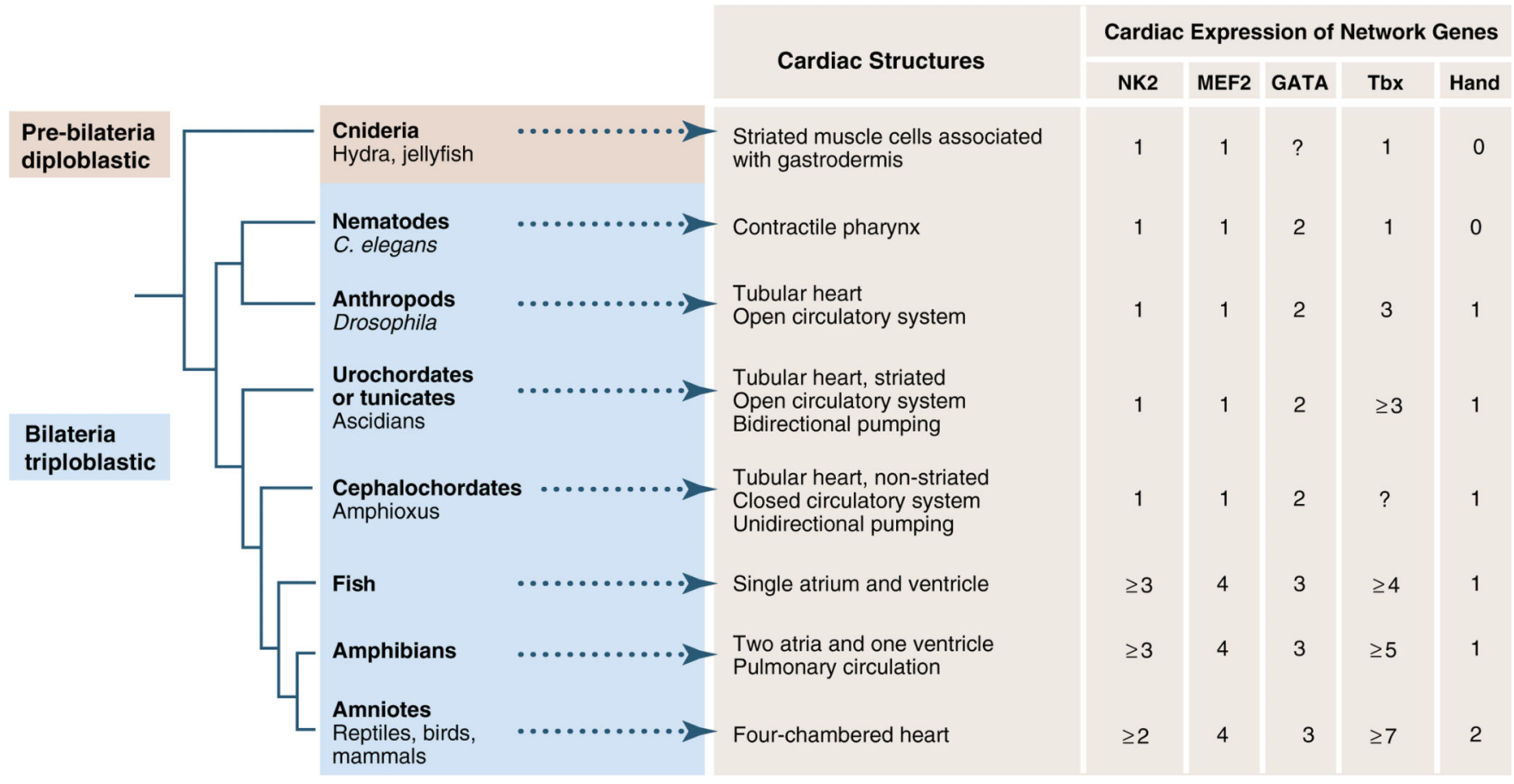

Fig. 1.

Evolution of the heart and the core cardiac transcription factors. The structures of the hearts of representative animals and their evolutionary relationships are shown. The numbers of cardiac regulatory genes, which are known to be expressed in the cardiac structures of each organism, are shown. 
A Simple chordates

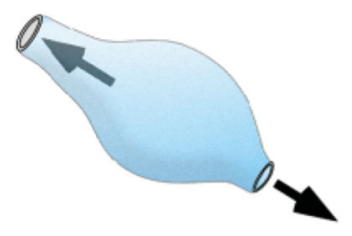

B

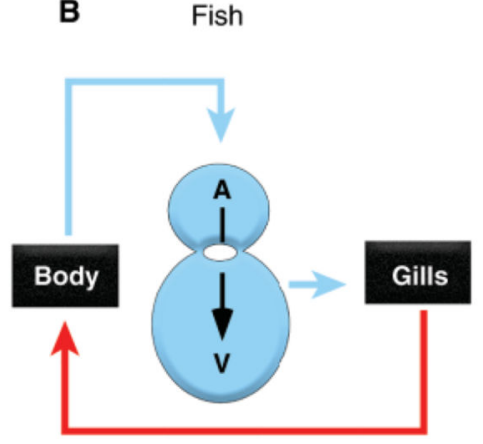

C Reptiles, birds and mammals

\section{Lungs}

RA LA
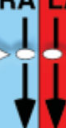

RV LV

Body

Fig. 2.

Simplified structures of different types of hearts, showing schematic diagrams of hearts and directions of blood flow. (A) Simple chordates have tubular hearts, some of which pump bidirectionally. The hearts of ancestral bilaterians probably had a similar structure. Fish hearts (B) have a single atrium and ventricle, whereas the hearts of reptiles, birds, and mammals (C) have two atrial and two ventricular chambers. Oxygenated blood is shown in red and deoxygenated blood in blue. 

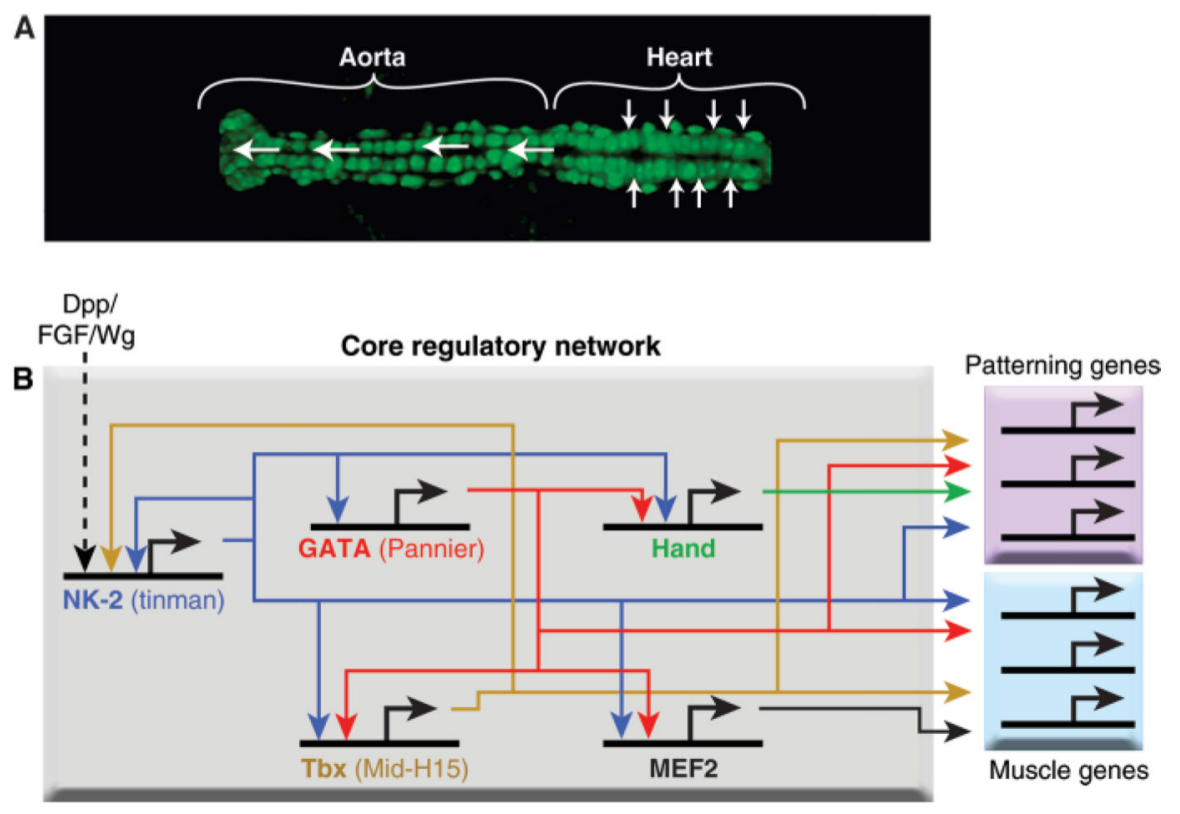

Fig. 3.

The heart of Drosophila. (A) The heart of a Drosophila embryo visualized by the expression of green fluorescent protein under control of the cardiac enhancer of the Hand gene.

Hemolymph enters the heart at the posterior end of the embryo through ostia and is pumped anteriorly. (B) A simplified diagram of the core transcriptional network of Drosophila heart development. 
A

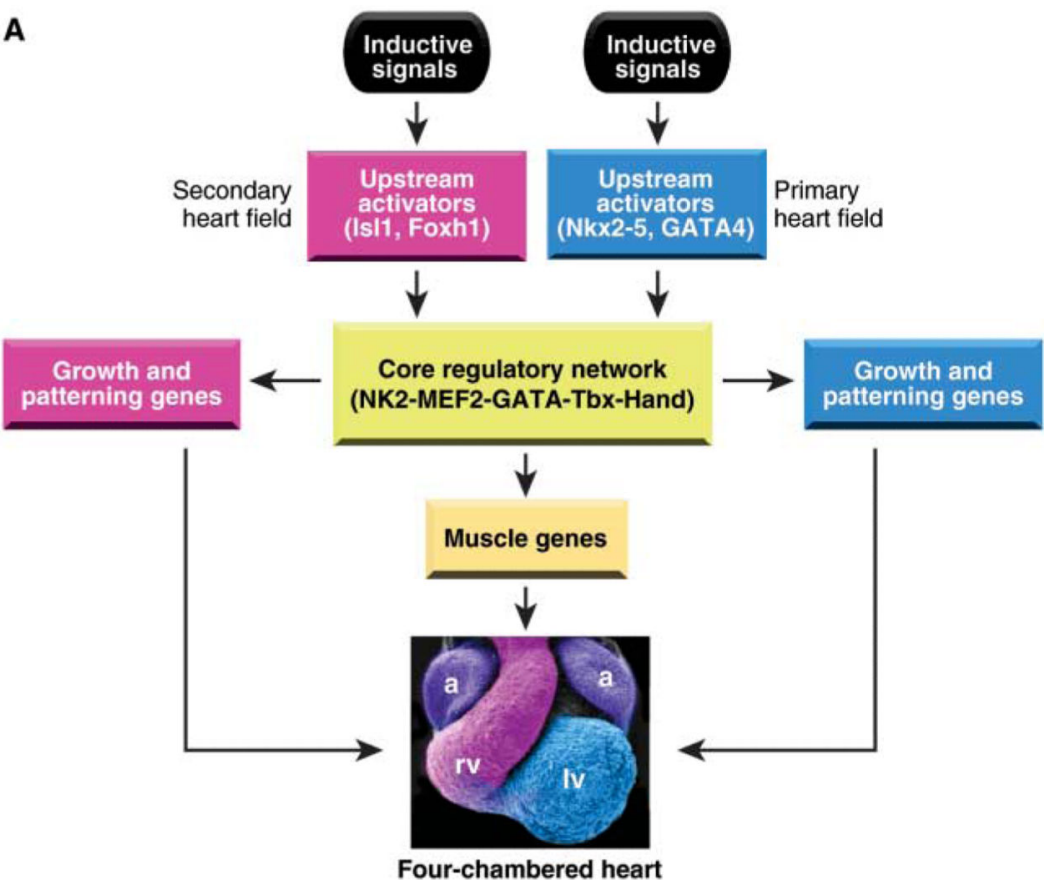

Inductive

signals

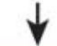

B

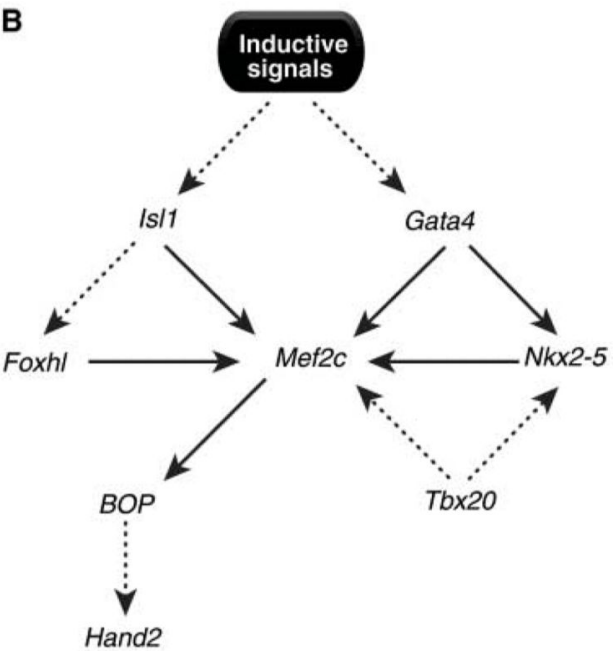

Fig. 4.

Schematic of transcriptional networks involved in mammalian heart development. (A) Inductive signals activate a set of upstream regulatory genes, encoding transcription factors, in the primary and secondary heart fields. The products of these genes activate the genes in the core cardiac network (NK2-MEF2-GATA-Tbx-Hand). Some components of the network, such as Nkx2-5, are also activated in the primary heart field in response to inductive signals. The core network genes cross- and autoregulate their expression and serve as the central regulatory network for the activation of muscle-specific genes and genes that control the growth and patterning of derivatives of the primary and secondary heart fields. The primary heart field gives rise to the left ventricle (lv) and portions of the atria (a), whereas the secondary heart field gives rise to the right ventricle (rv), portions of the atria, and the outflow tract. A scanning electron micrograph of a mouse heart at embryonic day 14.5 is shown at the bottom. Derivatives of primary and secondary heart fields are shown in blue and pink, respectively. The atria, which are derived from the primary and secondary heart fields, are shown in purple. (B) Regulatory interactions among cardiac transcription factors in the secondary heart field. Solid lines indicate direct transcriptional connections, and dotted lines indicate connections not yet shown to be direct. [Adapted from (9)] 